\title{
Googling the Anthropocene Fractal Media and Ecology
}

\begin{abstract}
The scars of humanity can be seen across the Earth. However, observing such ecological violence and their implications often requires finding the right perspective, moving beyond the spatial and temporal limits of individual humans. This article builds on discussion of the Anthropocene as a term and the anthropocentrism it implies to critique the relations between humanity, technology and ecology through posthuman perspectives. Focusing on Google's widely available tools, its problematic relation to the environment as a company, and critical interventions by media artists Mishka Henner, Paolo Cirio and Geraldine Juárez, the article examines technologies that enable a 'posthuman' position from which to view the fractal activities of humanity: Google maps and Earth; Street View; and the Google search engines. Fractals are offered as a mode of assessing the self-similar processes of mediation that define not only humanity's scalar expansion but also its shift into informational dimensions and the virtualisation of ecology.
\end{abstract}

Keywords: ecology, posthuman, technology, Google, Anthropocene, visualisation

\section{Introduction: a technological species, a technological world}

The Earth is a large object. Specifically, it is far too large for a single human to perceive. As a collective species, however, humanity has apparently mastered the Earth, spreading across its surface and leaving its marks throughout history and into the future. From the earliest cities to the constant expansion of the contemporary built environment, even the ruins of civilisation outlast the individual human or the culture that created them, creating an archaeology of human achievement and ecological destruction. These marks, these scars on the surface and oceans of the planet, include not only the actual structures created by humans in which to live, work, worship, or relax, but also the aftereffects of human activities. From farming through fossil fuels and radioactive materials to technological waste, this gradual build-up of the impact of humanity's expansion has reached a point where it is becoming recognised as a new geological age. While the individual human is a mere speck on the landscape, the Anthropocene marks a perceived shift in the lifespan of the planet. The planet is so large, so long-lived, so beyond an individual's ability to behold, that we can only view the full extent of the Anthropocene through the mediating lens of technology. As Morton writes:

Computational power has enabled us to think and visualize things that are ungraspable by our senses or by our quotidien experience. We live on more timescales than we can grasp...We are faced with the task of thinking at temporal and spatial scales are unfamiliar, even monstrously gigantic' (Morton 2016: 25).

Enter Google, the ultimate virtualisation of the universe, subsuming the material ecological impact of its server farms, network infrastructure and device manufacture with the 
informationalisation and categorisation of all things. From its origins as a search engine, Google has always sought to bring information of any kind directly to the human user, to revolutionise our access to the information age. This process establishes not only an epistemological dominance concerning what information is given a more prominent place in the algorithm's ranking, but takes on an ontological aspect of bringing every facet of the universe known by humanity into one connected entity, a gestalt memory of the species digitised and made accessible (at the cost of contributing one's own data). This virtualisation of human experience extends beyond merely its digitisation and computational manipulation. Google has used its technological innovations to insinuate itself throughout the global collective human consciousness, evidenced by its linguistic repositioning as the goto verb for acquiring knowledge. Google allows us to perceive more than our own experience, representing the full cybernetic potential of humanity in its extension through written language and now digital technology. This relation between technology and humanity is vitally important. More broadly, technology is both the means by which we can view the Anthropocene and the means through which it has been brought into existence. Google as a company embodies this dilemma. There is a problematic relationship between the startup ideals of the company, the progress of its marketing rhetoric, the business needs of the corporate entity, and the monopolisation of knowledge. Even within its technological innovations, Google displays a tension between its tightly-controlled proprietary search algorithms and the plethora of accessible or open source resources it offers to the entire world. More importantly, the direct ecological impact of using these tools highlights the risk of solutionism inherent to the dominant sociopolitical structures surrounding technology. This tension is echoed in humanity as a whole: the difficult task of comprehending, and taking collective responsibility for, the effects and inequalities of our own expansion.

In response to this dilemma of viewing the scale of humanity's impact on the Earth, this article will offer a new mode of viewing ecology in terms of fractal media: scalable perspectives on the self-similar behaviours of technologically-mediated human activity. Google will be examined as a set of tools that enable individuals to view the Anthropocene beyond the limits of the singular human and its constraints in space and time. Googlemaps and Street View will be assessed for their potential in enabling humans to understand the connections and implications of human activity as a whole. Artistic interventions in these tools will be discussed in their attempts to explicitly display ecological concerns through Google's technologies as well as their critiques of human expansion and the effects of the Anthropocene. These effects will also be considered in relation to Google as a company, the ecological impact of its technological infrastructure and its monolithic role in controlling the broader culture of technologically mediated ecology, information and the future of humanity. The article will conclude with a consideration of the role of posthumanity in beholding and critiquing the Anthropocene.

\section{Anthropocene, posthuman, fractal media}


As a term, the Anthropocene remains contested but not new. However, 'as a politically infused geology and scientific/popular discourse [the Anthropocene] is just now noticing the extinction it has chosen to continually overlook in the making of its modernity and freedom' (Yusoff 2018: loc.115). The geological community's ongoing debate over whether it should be recognised as a separate age and, if so, what the start date should be (ranging from the earliest agriculture to the industrial revolution or the mid-twentieth century) detracts from the urgency of climate action. Regardless of its formalisation and official geological acceptance, a Geological Society report (2014: 17) emphasised that the Anthropocene carries value across disciplines as an expression of the impact of humans on the Earth, a reminder of the long timescales at which this must be understood, and a tool for framing responses to this ecological impact. Outside of scholarly debates, what is labelled the Anthropocene has already been happening, it has just not happened equally across the world. The Anthropocene, like Google, represents the idealisation of material reality within a particular anthropocentric mode of thinking. It appears as the illusion of mastery by human consciousness and its desire to expand over the actual objecthood of the Earth as a distinctive entity. However, the Anthropocene must be understood not only as the virtualisation of ecology but, from this, as a problematisation of humanity. Haraway's (2016) critique of the Anthropocene is instructive here for its links to capitalism and power, its human exceptionalism and anthropocentrism, its individualism, its exclusion of divergent or marginalised perspectives, and its tendency towards defeatism. Instead, she proposes viewing the Anthropocene as a boundary event (2015: 160), a turning point, an opportunity for persisting with thinking about ecology and creating new futures. This echoes concurrent geological developments whereby the boundary itself is suggested to be distributed in time (Edgworth et al. 2015). Here we shall therefore understand the Anthropocene as a moment or process of realisation by humanity, not a definitive break or temporal division. The Anthropocene is a problematisation of humanity's relation to the Earth, a tool for highlighting anthropocentric perspectives and for thinking otherwise about the future of our species and the planet with which it is entwined.

The fact remains, however, that the individual human, unaided, cannot perceive the entirety of the Anthropocene that expands spatially and temporally far beyond the possible understanding of a single person. In order to conceptualise the human as an individual and species, and to diversify what it will become, we will need to expand our powers of perception and thought beyond the conventional frameworks of what we consider human. To change our relation to ecology, to overcome the Anthropocene, we therefore require new, posthuman modes of viewing ecology in relation to these technologically mediated forms of humanity and posthumanity as a series of connected practices between individuals, cities, countries and civilisations. The conception of posthumanism employed here builds on Braidotti's (2013) definition of critical posthumanism. Rather than emphasising the scientific technological considerations themselves, it is important to examine the ethical, relational and critical diversity of posthumanism as a mode of (re)thinking the nature of humanity. As with the Anthropocene, here the posthuman is a problematisation of the human as a problem. As Hayles (1999) would suggest, it is a new 
cognitive framework concerning informationalisation and the process of deconstructing the human. Braidotti's version of the posthuman is an expression of becoming, of nomadicity, and of collectivity. It is anti-humanist and anti-anthropocentric. It therefore aligns with Haraway's consideration of the generative effects of posthuman in conjunction with moving beyond it into 'compost'. We build on this here to consider two forms of 'post'-human. We are looking for perspectives 'after the human' in terms of the technologically-mediated (and therefore never neutral) expansion of our frameworks of thought and open possibility, as well as thinking 'after the human' in the literal sense of what happens when we remove ourselves from the (environmental) equation. The posthuman approach used here, then, is an active removal of the current ways of thinking the human subject while acknowledging that it is inscribed as a stain within reality, seeking new perspectives from which humanity can view itself simultaneously as present and absent, individual and collective. As Braidotti implores, critical posthumanism should move beyond anthropocentrism by embracing a nature-culture continuum of complexity and interconnection between self and other.

One method of bringing complexity and apparent chaos into a machine- and human-readable format across multiple scales is the fractal. A somewhat open term from mathematics, it has been applied to almost all areas of study from matter to geology to biology to human behaviour. While a set of rigorous mathematical tools, fractals lack rigid definition, with multiple different types and applications. Instead, they tend towards a number of key characteristics which can provide a useful conceptual toolkit for understanding scalable processes in technology and culture: detail on arbitrary scales; irregularity beyond description by conventional geometry; self-similarity (exact or approximate); a fractal dimension larger than its topological dimension; a simple definition or recursion (Falconer 2014: xxix). For the present discussion, the most useful characteristics relate to scalability, complexity, and increased dimensionality beyond the physical. Further, while a useful method of approximating (and thereby coming closer to understanding) chaotic structures, the fractal is an unnatural framework, an imposition of human rationality onto the complexity of the universe that would otherwise remain incomprehensible to humans. For this reason it is an instructive process with which to negotiate the limits of human thought concerning its relation to external systems such as ecology, in contrast to the inherently quantised, mediated, constrained realm of technology. Fractals allow us to view sets of information, collections of infinite points within boundaries, and the relations between points and between scales, but in doing so they always enact a form of lossy compression on reality. The reductionist collapse of anthropocentrism can be converted into a critical posthuman expansion; even within the bounding of a fractal set, the recursion of scale opens up infinite possibilities for rethinking our current situation, escaping the virtual boundaries of humanity by dislocating our perspectives into alternative scales in conjunction with radically different other(s). This echoes Braidotti's (2013: 102) call for a reinscribing of the posthuman within webs of power relations at all scales, in order to instantiate qualitative change. A fractal model thereby encourages us to 
view relational aspects of posthuman and ecological systems, and the interconnectedness and collectivity that will be required to overcome the impending environmental disaster(s).

A fractal model acts at the levels of part, assemblage and whole, incorporating geological, biological and informational distributions of activity. We cannot study ecology without studying ourselves: both within the systems and from the external position of viewer. Scaling our perspective is not only a matter of spatiotemporal size but also of epistemic type: of the system, of the observer, and of the observer-in-the-system. In relation to the big data that expresses such relations, fractals also highlight the usefulness and importance of visualisation when analysing large quantities of information that are conventionally only readable by machines. In conjunction with their technologies, humans can understand complex data through its aestheticisation and transposition into a human-readable format at the conceptual level. This connection between technology and humanity into the visual and artistic field highlights the importance of critical posthumanism in transcending the limits of a destructive and exploitative relationship with the environment, as well as the potential limits and benefits of open access to information and the tools with which to understand it. It is with this 'beyond ourselves' that humanity must view ecology and the self-similar impact and power structures of human activities across the planet, in increasingly fractal dimensions. In the wake of human mastery and destruction, the Anthropocene is arguably an era of post-ecology. Thus to view post-ecology we must take a posthuman view of ecology in order to confront the Anthropocene beyond the limits of conventional human perspectives. Taken collectively, mediated through technologies such as those provided by Google, and understood in relation to the constraints of this mediation, the fractal expansion of humanity can achieve such perspectives that are necessary to perceive and transcend the Anthropocene and the destructive limits of our species.

\section{Space: Google Earth}

Even the name 'Google Earth' demonstrates the informationalisation of the planet, with its load screen that measures progress as a proportion of the years of the Earth, or the amount of rock, water, atmosphere, sand or trees it contains. When the whole Earth is 'googleable', Google begins to take a certain level of ownership over the material object that constitutes humanity's spatial home. When one can manipulate the planet, soaring above the landscape at the click of a button, bringing new views of previously unknown or inaccessible places into our everyday experience, Google enacts an extreme virtualisation and unprecedented level of control over the Earth itself. With its reach extending across the physical globe and beyond into abstract datafilled dimensions, Google has become one of the most powerful mediators of humanity's relation to the universe. However, tools such as Googlemaps and Google Earth have been made freely available (if one ignores the somewhat abstract or speculative 'cost' of information tracking, the elusive revenue generated by targeted advertising, and Google's ultimate control over access), allowing each human to control their own interaction with the planet. This embodies the posthuman fractal perspective, for we can now self-determine the level of detail at which we 
perceive the Earth. We have gained the ability to zoom in and out, bringing new perspectives to life like moving through fractal imagery, generating the view on the screen with each click, scroll or swipe. Indeed, Google Earth adaptively loads image resolutions relevant to the current level of zoom, enacting a fractal motion of detail that shifts with the scale of the user-controlled perspective. We can navigate instantly across the world with urls, overlay our perspectives with hypertextual cartographies, and view 'parallel worlds' together in different windows to compare technological and ecological impact in radically different geographical areas. There is a posthuman agency and critical potential at work here. Yet this process treads a fine line of responsibility and engagement, with later versions of Google Earth offering a 3-dimensional flight around the world filled with interactive stories that, by the engineering manager's own admission, are 'more of a consumption experience' (Sean Askay in Leasca 2017). In the desire for ever more knowledge of the planet, our physical consumption of its resources is mirrored in the digital realm. A fusion of big data and aesthetic critiques are therefore required to present the wealth of experiences in a critical light that can enable Google Earth as a tool for viewing and reassessing the Anthropocene and its effects.

Applying these alternative or uncommon views of the Earth to issues of both ecology and technology has become the task of artist Mishka Henner. Henner uses satellite imagery (akin to that provided by Google), in order to construct critical aesthetic framings of specific instances of human ecological consequence. Known in part for his work Astronomical - a multi-volume book depicting the solar system in a human-readable scale (albeit mostly consisting of black pages representing the emptiness of space) - Henner plays with scale and the aesthetics of satellite imagery to push the limits of human perspectives and their links to ecology, space and information. The exhibition poster for Search History depicts imagery of Mars, and is described as 'exploring a series of diverse themes and challenges confronting the world today. Memory failure, oversight, truth and lies; A collection of signs, symbols, and landscapes of the $21 \mathrm{st}$ century' (Airspace Projects 2017). These concerns permeate Henner's work and its ecological mission, exposing the virtualisation of the landscape with its overlays of information and mediation by technology. The use of satellite imagery, a device that appears in many of his series, becomes conceptually removed from the way it appears in Google's tools. The shift into artistic practice enacts a particular aestheticisation of the images, a particular framing that is of interest to a fractal model of subjective perspectives. Rather than the specific selection of quantised views we are offered in Googlemaps or Google Earth, the zoom level and framing chosen by Henner is always an intentional aestheticisation of its subject matter. The images are self-similar in their (re)presentation as 'complete' images of specific sites, common across scales and demonstrating the familiar forms of human activity the world over. This combination of a painterly frame and the technologically enabled images taken from beyond the bounds of conventional human existence takes on a fractal point of view, able to shift its perspective to the view required to apprehend and comprehend the position and functioning of what in effect are 'portraits' of humanity (including its livestock and machines) in relation to the Earth. 
The series of images entitled Feedlots displays the impact of the (American) meat industry, including the infrastructure used to reduce maturity (read: slaughter age) from 5 years to 18 months. This process includes hormones, antibiotics and feeding, enabled by large scale production facilities. The regularity of the fields or lots in the images is often mixed with the more organic structure of watering holes, here reminiscent of infected cells in a collision of ecology with human virtuality, two forms of fractalisation in the aesthetic reframing of the subject matter. We see the effects of converting nature into information, the human process of pattern-recognition that re-patterns the landscape into quantised zones of ownership. Henner (2015) writes, 'I don't just see gigantic farms, I see an attitude toward life and death that exists throughout contemporary culture. These images reflect a blueprint and a horror that lie at the heart of the way we live.' He is turning human and posthuman technologies back onto themselves in order to highlight the violence of anthropocentrism and human exceptionalism. The blackened scars that mark this attitude towards the death of the planet as a whole can be seen in further series: Fields, Eighteen Pumpjacks and Libyan Oil Fields. These images show the sites of the oil industry with their geometric buildings and equipment standing in stark contrast to the desert and otherwise deserted landscape, and the streaks of smoke across the satellite imagery display the chaotic fractal spread of callous, inward-looking human activity. 51 US Military Outposts takes a slightly different angle. There are some industrial and pollution markers here, but the impact is felt in the implied infrastructure backing the military industrial complex, while the juxtaposition of the outposts against otherwise idyllic locations emphasises the ecological impact of internal human conflict and the factional and fractional nature of our networked species. Into the digital realm, Dutch Landscapes highlights the attempts by human social structures to exert control over information by showing locations that were forced to be blurred out or pixelated on resources such as Googlemaps. These sites of human (national) importance, such as palaces or military sites, display the reactionary censorship of the new posthuman perspectives that move purposefully beyond traditional state machinery. There is a virtual battle taking place between state and capitalist claimants over information, access and control. In order to open the traditional constraints on humanity's point of view, the posthuman potential for the aestheticisation of space requires an openness of technology. While fueled by consumption and data collection, Googlemaps and Google Earth offer accessible tools with which to promote a posthuman and post-ecological view of humanity and its environmental debt.

\section{Time: Street View}

Where Googlemaps and Google Earth enact a spatial expansion of human perception and cognition, Google Street View remains embedded within a more familiar mode of interacting with the world. The movement allowed by the Street View interface appears more as a limit to the movement of a human down a street. We can only shift from one quantised position to another, along set paths defined by the streets themselves and the routes the Google cameras have previously travelled. What Street View offers instead is a distortion of time, the ability to 
remediate and partially manipulate our relativistic and subjective frames of reference against our technologically mediated movements. We retain the virtualised and hypertextual movement but are presented with an image embedded within the world yet clearly frozen in time. This fractal distortion of a familiar stroll down a street creates new temporal aesthetics for the posthuman flaneur. It also allows us to view an impossible historical perspective, to be in places we could not possibly have been at that time, particularly with the simultaneous experience of different spaces and different times. The Street View tells the viewer the date of the particular view, and includes the option to travel back in time to previous versions. This mode brings to the fore the evolution of human civilisation, emerging in virtual fractal patterns as buildings appear and disappear in a quantised timeline locked in to specific previous experiences of the humans who took the pictures. This belies a deeper implication of human agency, for it is only along the marks of urbanisation that the Street Viewer can travel. We become confined to the linear scars our species has left upon the face of the Earth, forever embedded within the legacy of the Anthropocene and the prior paths well-travelled by previous humans. To enter Street View is to confront the history of humanity's impact upon the planet, and the digital flaneur here is condemned to marvel at the overwriting of the environment by the virtualisation that constitutes the Anthropocene.

The tension between individual human and collective humanity is further enhanced by Street View. On the informationalised global scale, the 'pegman' avatar appears as an abstract placeholder for all of humanity, a common aestheticisation as data point. On some level our experiences in Street View are all the same, always already predetermined and over-mediated by the structures of the technology and the constraints of the tool itself. It is also notable that any form of avatar is removed from the screen in the actual Street View itself. Combined with the absence of the observer from the image, this shifts the individual spectator's posthuman perspective into a fractal position of detached observer, becoming able to simultaneously view the world and their mediated position in it. Against this semi-abstracted meta-medial position, there remains a human element to Street View, a collective embracing of the aesthetic value of individual experience. Where roads end, we see instead the impact of human exploration through its historical structures and beyond. For example, even though Egypt and China (for political reasons) and Antarctica (for lacking infrastructure) are not covered by Street View on their road systems, the frozen paths of individual humans trekking around the Pyramids, along the Great Wall and around research stations still offer Street View data. This crowdsourcing approach makes use of aggregated individual human perspectives to construct an atemporal posthuman collective point of view. Humans (through and for Google) continue to conquer the world physically and digitally to bring the planet under their collective control, while celebrating and aestheticising the individuals and their experiences that make it possible.

Turning the posthuman perspective of Street View directly onto the relation between humans and their urban environment, Paolo Cirio’s Street Ghosts project brings the collective species' 
biological memory into view within the technologically-mediated framework. Images of people immortalised on Street View are pasted onto the physical locations before being re-mediated back into digital images and pinned on a Googlemap. Between the atemporality of data, the temporality of individual humans, and the various forms of marks left by the Anthropocene, the project engages with a critique of the posthuman and its medium. As the Cirio (2012) writes, 'the public display of this biopolitical surplus from Google's value-harvesting campaigns - the people aren't supposed to appear in the pictures, but they do - appropriates their aesthetic and political value, as opposed to the commercial.' This is a rejection of the removal of the individual observer, embedding the human in the image and entrenching the fractal dislocation in space, time and data of human subjects, spread further across the world via the technologies it critiques in order to enmesh even further perspectives of posthumanity. 'Posthuman' here refers not only to technological enhancement but also to the transition of the individual to a ghost in the form of atemporal virtualised data. The repeated processes of removal at work in Street Ghosts shows a technological recursion, going down-the-rabbit-hole of human agency and mediation: the original humans are removed from the physical locations by their own movement, but fixed in place as data on Google's servers; the identities of the individual humans appearing on Street View are removed through blurring of faces to comply with data protection regulations; the fleeting existence of the figures on screen are removed once we leave the location or page; the digitally fixed characters become temporal in their impermanent physical replication; the physicality is once again removed in the fixing of abstract hypertextual pins denoting their locations on Cirio's own world map. Yet across these different forms, the same processes of the mediation of information (about humans) persist, self-similar across media. This framing and reframing, the objectification of humans in Cirio's work, highlights the processes that are constantly shifting the scope and perspective of/on the individual human in the broader context of the collective fractal species. His work embodies the critique by Ingraham and Rowland (2016) of Street View as the realisation of ubiquitous surveillance and biopolitical control. The critical role of Cirio's Street Ghosts is to emphasise the difficulty of removing humans from the equation, we always persist in the anthropocentric, capitalist systems that define the problem of the Anthropocene. It is not only the universe but also each other that we compress into data to be stored, aggregated, sold and consumed. From the critical position that highlights these processes, we can perhaps contextualise and re-mediate our networked processes of thought and memory, whereby posthumanity emerges as a collective consciousness with fractal agency. Through the persistent processes of mediation and the recursive self-similar relation between humanity and technology, we are constantly at risk of returning to the Anthropocene. We require ever new ways of critically visualising our ecological impact if we are to take responsibility for our destructive behaviour towards the planet and ourselves.

\section{Data: Google}

If the Anthropocene is the virtualisation of the universe through the creation of human-made technologies, then the transition into the digital age has signalled a duplication of this process. 
Where 'virtualisation' is taken in the broader understanding of idealisation, approximation and the replication of an aspect of the universe in an informational format within the collective consciousness of humanity, the shift into computational media represents the reification of data. The already fractal constructs gain a new level of fractal dimensionality in digital technology. Informationalisation does not translate real objects into ideal ones, but rather adds an extra layer of manipulable understanding and substance to spatiotemporally distributed objects. It is this substance that Google gathers, stores, arranges and sells in its billion-dollar business model: the universe as data. The fulfilment of the virtualisation that characterises the Anthropocene is the full transition of the material world into the substance of data, a twisting back on itself that entrenches the epistemological frameworks of posthuman consciousness as a basis for future reality. It is here that the ontological responsibility of humanity becomes most evident, in the doubled ownership of the planet as data to be manipulated. Here too lies Google's questionable role, for there is a techno-political and epistemic aggression occurring in the crawlers (often called robots or spiders - and we see here the Silicon Valley appropriation of Haraway's (2016) critical use of tentacular entities) that actively scrape information from the web, consuming the collective network and claiming for its own. This process appears to be for accessibility and usability of information, but it is underpinned by the economic value of advertising and data tracking. If digital technology enables the collective externalisation of thinking and remembering that hails a shift towards posthuman consciousness, then it is to the mighty server farms and data centres of companies such as Google to whom we hand over parts of the power of thought.

Yet the datafication of our environment also possesses positive, constructive, critical potential for a more self-aware posthumanity. The algorithmic perspectives on reality, while seemingly reductive, are not only growing ever more complex but also allow us to highlight the processes of control and mediation which we enact upon the world and ourselves. Algorithmic bias and feedback loops are important to acknowledge, and can highlight the self-similar and chaotic patterns of individual and collective human thought that lead to ecologically destructive behaviours. Google, as the principal search engine and data-gatherer on the planet, offers us such tools with which to assess the culture of ecology. Table 1 shows the number of Google search results (Google 2018a) for words relevant to the present discussion. The list displays some of the relative cultural positions of technological and ecological terminology, along with forecasts of their economic impact for Google Adwords (Google 2018b), Google's revenue stream. While far from a detailed corpus analysis of internet content or Google's secretive proprietary search algorithms, a few points stand out. Google itself, and technology in general, both feature more prominently than nature, the environment or earth. While the generic and hopeful 'future' features highly, the specificity and responsibility of 'Anthropocene' and 'posthuman' are tiny footnotes by comparison. It is perhaps worrying to note that within the past ten months 'pollution' has overtaken 'humanity' in the number of hits received. The results also highlight that despite Morton's (2009) assertion of the need for 'ecology without nature', the persistence of 'nature' is clear. It is notable that the top interest in 'ecology' from Google searches occurs in 
Ethiopia, while 'nature' receives significantly greater interest in more economically developed, post-industrial countries (Google 2017). The Adwords forecasts echo many of these relationships, but the somewhat more abstract 'nature', 'future' and 'humanity' display less economic value than the more concrete 'environment' and 'earth'. The nostalgic image and more specific objecthood perhaps provides a link between the easily human-comprensible and an idyllic or delusional self-image. The speculative value at work here signals Google's ability to perceive trends at both extremes of temporal scales, its adaptive algorithms adjusting to micro and macro changes in the social environment. Through the aestheticisation of this big data, Google can bring to our fingertips a clear and human-readable overview of our position in relation to the plethora of interconnected networks and systems and their ecological implications, as well as their relation to humanity as a whole. Comparing even basic data with theories and experiences (which of course must also be found through a Google search), we can reveal a great deal about the cultural role of terminology and its proliferation throughout digital culture as a whole.

[insert table 1 near here]

The role of Google goes beyond purveyor of information, for the company has come to take a prominent place in determining our technocultures and approaches to information. Google's near monopoly over information defines the framing of humanity's agenda, and the structures of presentation and representation it controls can be felt in ecology. One such example is the Google doodle, the friendly welcoming graphics and interactive pieces that adorn the search engine's homepage to signify noteworthy events, anniversaries and ideas. There have been many Google doodles with an ecological emphasis, but of particular interest here is the Earth Day doodle, which has run annually since 2001 and is now featured as widely as the search engine is accessible. According to Google (2016) Earth Day 'may be the one day that transcends culture, language, and tradition', and the 2017 doodle was featured in TIME magazine for its 'urgent message about climate change' (Gajanan 2017). Information here becomes profoundly political, and the manner in which it is presented, aestheticised and culturally integrated displays Google's reach and potential power for change. However, embedded within this public relations exercise remains the questionable role of Google as contributor to ecological damage and mediator of our access to knowledge of it. They are positioning themselves as the shining gatekeepers to an imaginary landscape, behind which stands a barren wasteland.

The self-similar exploitation of cultural and ecological resources under the unifying umbrella of 'data' is compared to colonial and neo-colonial bio-prospecting in the virtual reality (VR) essay PROSPEKT by Geraldine Juárez. This work highlights Google's displaying of cultural objects and heritage from around the world, made available online for free, as an act of claiming ownership and the assertion of control over the manner of future access. Physically located within a tropical greenhouse, the VR event enacts a 'a viewpoint, a wandering ogle that 
examines, sorts and determines meaning and value' (Juárez in Debatty 2019), locating Google within a colonial and capitalist history of encyclopaedic mediation of the world. This echoes the need for diversity in countering the dominant racial and colonial framing of the Anthropocene which:

proclaims a sudden concern with the exposures of environmental harms to white liberal communities $[. .$.$] in the wake of histories in which these harms have been knowingly$ exported to black and brown communities under the rubric of civilisation, progress, modernization and capitalism. (Yusoff 2018: loc.115)

This 'selective perspectivism' is a retelling and appropriation of reality by technology and capital: culture in, toxic sludge out. In PROSPEKT, with the aim of demonstrating that 'organising information is never innocent', Juárez uses VR to perform a 'technical gaze' outside of time and space, and thereby 'presenting the world-as-an-endless-digital-exhibition' (Juárez in Debatty 2019). The work highlights the datafication of ecology and society in equal measure, the full virtualisation of the planet by Google and the final fulfilment of the Anthropocene. What Juárez offers us is a critical counterpoint, providing technologically-mediated perspectives not into the data itself, but into the processes of framing that define the new systems of power, access and control that Google now leads.

Prates (2017) defines the Anthropocene as the era when biosphere disappears as externality with corporate sustainability as a perverse response alongside a consumptive collective denial. What is required, he argues, is a radical subjectivity emerging from the truth of the Anthropocene as a destructive event, in order to generate new political visions of the environment. Yet we must remember also Haraway's emphasis on this event of the Anthropocene as only a boundary, and ensure these new subjectivity's follow Braidotti's posthuman ethics. As one of the largest and most influential corporations in the world, Google remains complicit in the impending ecological collapse, even as it offers posthuman structures of reconceiving our role in the environment. Google continues to make major contributions to specific cases of positive ecological impact: Google Earth has been used by Australia's Department of Environment and Conservation to discover the formation of rare coral reefs; Streetview cars have been fitted with, air pollution sensors to create maps of forest loss; AI has cut cooling energy costs in data centres by $40 \%$ through increased and adaptive efficiency operating at timescales far faster than humans could react (Evans and Gao 2016). Through Google, the fractal posthuman can expand its reach spatially and temporally in both directions of scale. However, it is not only content that we consume, for Google's role as a technology company remains bound to issues such as energy usage. The cooling energy required for data centres remains excruciatingly high, particularly in technocultural centres such as California. While Google aimed for carbon neutrality and 100\% renewable energy (a project titled ' $\mathrm{RE}<\mathrm{C}$ ': renewable energy cheaper than coal), the ever increasing need for power led to the abandonment of the renewable ideal. Their impact is instead apparent only on human-readable scales such as the visible use of goats to trim the grass on their famous Mountain View campus, a visual symbol of how the company wishes to be perceived. In 
their report to the Carbon Disclosure Project, a nonprofit assessing companies' awareness of the impact of climate change, Google wrote: 'if customers value Google Earth Engine as a tool to examine the physical changes to the Earth's natural resources and climate, this could result in increased customer loyalty or brand value' (Google in Flavelle 2019). This highlights the overwriting of technology as a cause of ecological damage in favour of corporate (re)branding as mediator of the collapse it is itself causing. Google's motto of 'don't be evil', echoed but intentionally more vague in parent company Alphabet's later motto 'do the right thing', has severe limits and highlights the ethical relativism of anthropocentric agency. Google is known for its visions of the future, but never specifies whose future. Many of Alphabet's subsidiaries focus on the future of humanity as an apparently homogenous concept, but a commitment to the future of the planet remains hazy at best. What is missing is a set of detailed ethical constraints on the application of Google's technologies. Rather than the small-scale, marketable activities that become clearly visible in Google's media presence, it is essential to maintain the fractal view of humanity and posthumanity if we are to perceive the Anthropocene and define the futures of ecology and humanity at all scales of space and time.

\section{Conclusion: posthumans for ecology}

Googling the Anthropocene is an engagement with new mediated perspectives that enable humans to view ecological information in new ways. This is allows individual human subjects to think in scales that are often beyond singular perception, and it signifies the convergence of material objects with their extra informational fractal dimension. Moving beyond physical spacetime, twisting our thoughts into those of the technologically mediated fractal posthuman, is a step through Engelbart's (1962) early cybernetic vision of augmenting human intellect and into what has previously (Benjamin 2016) been described as a cyborg posthumanity between physical and digital modes of thinking. On the other hand, Googling the Anthropocene could be taken to mean creating (or at least contributing significantly to) the ecological situation humanity has set in motion. While it allows us to view the technological impact of humanity and its products, Google itself remains one such product, and a complicit part of anthropocentric ecology. It is Google and other technologies that generate the requirement for a contorting of perception, abstraction and aestheticised representation in order to understand ecology. When the situation is pushed beyond the Earth itself into spatiotemporal and abstract dimensions beyond conventional knowledge, into the mysterious and almost mythical realm of big data and machine learning, the posthuman technological structures create a system of post-ecology.

Yet Google's tools, and the lessons of its dilemmas as an entity and actor, allow us to visualise and highlight trends and flaws in the fractal collective agency of humanity and its ecology culture. It allows us to take critical posthuman perspectives on the mediated relation between humanity, technology and ecology. Extending human perspectives with new and enhanced modes of pattern recognition creates opportunities for critical representations of ecology at multiple scales of space-time. These new structures can be experienced simultaneously in a 
digitised fractal reality. If the future of the planet is to remain open, humanity will need to use technology to balance and critique its own opportunities and risks, to guide its own development and understanding. More critical than viewing information about the Anthropocene is perceiving the fractal limits of anthropocentrism. To save the earth will require first changing ourselves. It should be noted, however, that this does not necessarily require saving humanity, for that would likely be at the expense of the planet. The environmental cost of technology should not exceed its use to combat the costs of the Anthropocene. Nor should it signal a return to a lost state of 'nature'. Rather, we are presented with an opportunity to recreate ourselves, to transform humanity into something new. This is the ultimate destructive and creative act of posthuman agency and aesthetics, and we must make sure that we are aware of the power that corporations such as Google exert over our access to knowledge, even as they bring us the future with a userfriendly interface.

\section{References}

Airspace. (2017). SEARCH HISTORY - Mishka Henner. Retrieved from http://www.airspacegallery.org/index.php/projects/search_history_mishka_henner.

Benjamin, G. (2016). The Cyborg Subject: Reality Consciousness Parallax. London: Palgrave Macmillan.

Braidotti, R. (2013). The Posthuman. Cambridge: Polity.

Cirio, P. (2012, September 15). Street Ghosts project. Retrieved from http://streetghosts.net/.

Debatty, R. (2019). PROSPEKT. Organising information is never innocent. We Make Money Not Art. Retrieved from http://we-make-money-not-art.com/prospekt-organising-information-isnever-innocent/.

Edgworth, M., Richter, D., Waters, C., Haff, P., Neal, C. and Price, S. (2015). Diachronous beginnings of the Anthropocene: the lower bounding surface of anthropogenic deposits. The Anthropocene Review, 2(1), 33-58.

Engelbart, D. (1962). Augmenting Human Intellect: A Conceptual Framework. Summary Report AFOSR-3233.

Evans, R. and Gao, J. (2016, July 20). DeepMind AI reduces energy used for cooling Google data centers by $40 \%$. Retrieved from https://www.blog.google/topics/environment/deepmind-aireduces-energy-used-for/. 
Falconer, F. (2014). Fractal Geometry: Mathematical Foundations and Applications (3rd ed.). Chichester: John Wiley \& Sons.

Flavelle, C. (2019). Corporate America Is Getting Ready to Monetize Climate Change. Retrieved from https://www.bloomberg.com/news/articles/2019-01-22/muggy-disney-parks-downed-at-ttowers-firms-tally-climate-risk.

Gajanan, M. (2017, April 21). Google's Earth Day Doodle Sends an Urgent Message About Climate Change. Retrieved from http://time.com/4751376/google-doodle-earth-day-tips-climatechange/.

The Geological Society. (2014). Geology for Society. London: The Geological Society.

Google. (2017, April 22). Earth Day 2016. Retrieved from https://www.google.com/doodles/earth-day-2016.

Google. (2017, April 20). Google trends. Retrieved from https://trends.google.com/trends/.

Google. (2018a, February 12). Google search. Retrieved from https://google.co.uk.

Google. (2018b, February 13). Google Adwords. Retrieved from https://www.google.com/url?q=https://adwords.google.com/ko/KeywordPlanner.

Haraway, D. (2015). Anthropocene, Capitalocene, Plantationocene, Chthulucene: Making Kin. Environmental Humanities, 6, 159-165.

Haraway, D. (2016). Tentacular Thinking: Anthropocene, Capitalocene, Chthulucene. E-Flux, $75,1-17$.

Hayles, K. (1999). How We Became Posthuman: Virtual Bodies in Cybernetics, Literature, and Informatics. Chicago: University of Chicago Press.

Henner, M. (2015, December 27). How the meat industry marks the land -- in pictures. Retrieved from http://www.latimes.com/opinion/op-ed/la-oe-marks-on-the-land-html-20151222htmlstory.html.

Ingraham, C. and Rowland, A. (2016). Performing Imperceptibility: Google Street View and the Tableau Vivant. Surveillance \& Society, 14(2), 211-226. 
Leasca, S. (2017, April 19). Google Earth Got A Stunning New Update Just In Time For Earth Day. Retrieved from https://www.good.is/articles/google-earth-earth-day.

Morton, T. (2009). Ecology without Nature: Rethinking Environmental Aesthetics. Cambridge, MA: Harvard University Press.

Morton, T. (2016). Dark Ecology: For a Logic of Future Coexistence. New York, NY: Columbia University Press.

Prates, V. (2017). The Anthropocene Diet: perversions of consumers facing the environmental crisis. International Journal of Zizek Studies, 11(1), 1-16.

Yusoff, K. (2018). A Billion Black Anthropocenes or None. Minneapolis, MN: University of Minnesota Press. 
Table 1

\begin{tabular}{|l|l|l|l|l|}
\hline \multirow{2}{*}{ keyword } & \multirow{2}{*}{ \# of search results } & \multicolumn{2}{l}{ Adwords forecast } \\
\cline { 3 - 5 } & & clicks & impressions & cost \\
\hline Google & & $6.1 \mathrm{k}$ & $119 \mathrm{k}$ & $£ 948$ \\
\hline technology & $10,630,000,000$ & $1.26 \mathrm{k}$ & $25 \mathrm{k}$ & $£ 345$ \\
\hline nature & $4,930,000,000$ & 614 & $11.5 \mathrm{k}$ & $£ 158$ \\
\hline future & $2,850,000,000$ & 250 & $6.76 \mathrm{k}$ & $£ 61$ \\
\hline environment & $2,270,000,000$ & 553 & $12.6 \mathrm{k}$ & $£ 306$ \\
\hline earth & $1,630,000,000$ & 628 & $7.55 \mathrm{k}$ & $£ 232$ \\
\hline pollution & $1,450,000,000$ & 247 & $6.29 \mathrm{k}$ & $£ 87$ \\
\hline humanity & $194,000,000$ & 20.8 & 368 & $£ 4.70$ \\
\hline ecology & $190,000,000$ & 58.5 & $1.21 \mathrm{k}$ & $£ 14$ \\
\hline anthropocene & $131,000,000$ & 17.2 & 567 & $£ 12$ \\
\hline posthuman & $2,110,000$ & 0.236 & 5.91 & $£ 0.21$ \\
\hline
\end{tabular}

Google search results (UK, 12/02/2018) and adwords forecasts (all locations, English, Google, 13/02/2018) for keywords concerning technology, ecology and posthumanity. 